\title{
D'Agrippa de Nettesheim à Montaigne: L'embarras des médecins devant l'origine de la semence
}

Par Jean Starobinski

On sait l'usage que Montaigne fit de l'ouvrage d'Agrippa de Nettesheim sur l'Incertitude et la vanité des sciences. Il y trouva, entre autres, ample matière pour renforcer les thèses sceptiques de l'Apologie de Raymond Sebond (II, XII) et l'argumentation antimédicale de l'essai II, XXXVII (De la ressemblance des enfans aux peres). Pierre Villey, dans son livre monumental ${ }^{1}$, et dans le volume de l'édition municipale où il indique les sources de Montaigne ${ }^{2}$, a signalé les nombreux emprunts, qui attestent l'étroite familiarité de l'auteur des Essais avec l'ouvrage d'Agrippa. Il est un rapprochement, toutefois, qu'il a omis de faire, sans doute par scrupule d'érudition: les deux passages qui me paraissent devoir être confrontés ne comportent pas suffisamment de similitudes littérales pour qu'il soit possible, en l'occurrence, de considérer Agrippa comme la «source» certaine de Montaigne. N'y ê̂t-il, toutefois, qu'une simple coïncidence, il serait important de la relever.

La critique de la médecine, dans le De Incertitudine, occupe les chapitres LXXXII et LXXXIII. Le premier de ces chapitres concerne le statut scientifique de la médecine; le second s'attaque plus précisément à la conduite des médecins. Au chapitre LXXXII, le premier thème abordé est celui du rang de la médecine parmi les arts: la question est loin d'être nouvelle; Pétrarque l'avait soulevée dans ses Invectivae contra medicum ${ }^{3}$... Montaigne, si peu intéressé par l'institution universitaire, ne fera pas état de cette «querelle des facultés».

Suit, chez Agrippa, un rappel très succinct des trois «sectes» qui se sont disputé la prééminence dans l'antiquité: «dogmatique», «empirique», «méthodique». Agrippa n'oublie pas de mentionner les Arabes, dont l'autorité a supplanté celle des Anciens. Montaigne, à son tour, tracera une rapide histoire de la médecine ${ }^{4}$, autant d'après Agrippa que d'après leur source commune, Pline l'Ancien (H.N.XXIX, 1).

Chez Agrippa, la polémique ne commence véritablement que sur un troisième point: l'impuissance des médecins à se mettre d'accord sur une explication exacte des grands problèmes de la vie et de la maladie. Vient en tout premier lieu la question du «sperme ou semence generative», que les 
Anciens n'ont pas su résoudre de façon univoque et satisfaisante. Les autres points sur lesquels les médecins n'ont aucune certitude sont: la cause des maladies, la digestion des aliments, et enfin, une question d'embryologie: le poulet prend-il sa croissance dans le blanc ou dans le jaune de l'œuf? L'embarras majeur, on le voit, c'est, pour Agrippa, l'origine du vivant: chaque philosophe a proposé sa réponse, et la question est restée indécidée. Telle est la crux qui condamne les médecins à une ignorance fondamentale. Comment comprendre ce dont on ne peut expliquer la genèse? A plus forte raison, comment agir de façon sûre quand on ne sait pas comment s'est formé le corps auquel on prétend porter secours. Le second objet d'ignorance, touchant la cause de la maladie, achève de rendre vaine la prétention de la médecine: si l'on n'a aucune notion de l'origine du mal, il est illusoire de vouloir lutter contre lui, car toute lutte efficace s'adresse à la cause... Agrippa, sur cette double question d'origine, se borne à un exposé doxographique: il laisse au lecteur le soin d'en tirer les conclusions.

Montaigne procède, à l'évidence, de façon bien différente. Le chapitre $D e$ la ressemblance des enfans aux peres, qui clôt le deuxième Livre des Essais, et qui dans l'édition de 1580 constituait la conclusion de l'ouvrage entier, est d'abord l'occasion d'un retour sur soi-même et d'un regard d'ensemble sur l'entreprise des Essais. Montaigne signale à son lecteur que, depuis dix-huit mois, il a subi à diverses reprises les atteintes de la pierre. Priorité est donnée à la relation de l'expérience personnelle: «J'entre des-jà en composition avec ce vivre coliqueux; j'y trouve de quoy me consoler et de quoy esperer». ${ }^{5}$ De loin, il avait redouté la douleur. A en faire «l'essay», il découvre qu'il en avait «plus de peur [qu'il] n’y [a] trouvé de mal». ${ }^{6}$ Il peut, lors des accès, garder bonne figure, et conserver une conscience entière. Il apprivoise ainsi la mort. Les préceptes de la philosophie stoïcienne lui apparaissent excessifs et inutiles. Il faut savoir faire sa part à la souffrance; il ne faut pas avoir honte de pousser des cris si la douleur nous en arrache. Montaigne concilie de la sorte, l'acceptation du mal et le pouvoir de la réflexion, même si la maîtrise de soi devient plus intermittente et précaire: «Je me taste au plus espais du mal»... ${ }^{7}$

Ces confidences de Montaigne sur son comportement face à la maladie ne concernent encore nullement la médecine. Elles nous préparent toutefois à mieux comprendre la polémique antimédicale qui occupera le reste de l'essai. Comment Montaigne s'y prendra-t-il pour passer à ce second thème, où, sans que les considérations personnelles disparaissent tout à fait, le débat prendra un tour plus général et plus épistémologique? 
C'est dans cette transition qu'intervient une argumentation dont on n'a, à ma connaissance, pas suffisamment souligné la similitude avec la stratégie polémique (beaucoup plus fruste, il est vrai) d'Agrippa: le défi lancé à la médecine choisit d'ouvrir le combat par la question de la semence.

Agrippa, avons-nous dit, se contente de la juxtaposition des opinions discordantes, à la manière de tous les doxographes. Il confronte les théories générales contradictoires par lesquelles les philosophes se sont singularisés. C'est le moment de citer le passage qu'il consacre à la semence:

Or combien que les sectes $\&$ factions des medecins soyent peu en nombre, si est ce qu'il y a aussi grande contrarieté d'opinions entre eux qu'entre les philosophes. Comme en ce qu'ils debattent du sperme ou semence generative avec leurs raisons sottes \& arguments de vieilles: car Pythagoras disoit que c'estoit l'escume du sang le plus pur, \& l'excrement de la plus pure \& utile nourriture. Plato que c'est une humeur coulante de l'espine du dos \& de la moëlle d'icelle, pour autant qu'à ceux qui usent trop souvent de la compagnie des femmes le dos et les reins deulent. Alcmeon que c'est une portion de la cervelle, pource aussi que les yeux font mal à ceux qui sont excessifs en cest acte, attendu que l'œil est partie du cerveau. Democrite dit qu'elle procede de toutes les parties du corps humain, \& Epicurus qu'elle est esprainte du corps \& de l'ame. Mais Aristote enseigne que c'est l'excrement du sang nourrissant, \& de la derniere digestion d'iceluy par les membres. Les autres ont opinion que c'est du sang cuit \& blanchi dans les genitoires par la chaleur d'iceux, fondés sur cette seule raison, que ceux qui sont trop aspres à l'œuvre de Venus au lieu de semence jectent gouttes de sang pur. En outre Aristote \& Democrite afferment que la semence de la femme ne sert de rien à la generation, \& nient qu'elles ayent germe aucun, ains seulement jectent une certaine sueur peculiere: Mais Gallien soustient que les femmes jectent semence, imparfaicte toutesfois, \& que tant celle de la femme que celle de l'homme ensemble forment le fruict. Au surplus Aristote veut que les corps des animaux soyent engendrés proprement de sang, \& d'iceluy immediatement nourris, \& que le sperme a sa generation du sang. Or Hippocrates au contraire dit que les corps des animaux sont premierement assemblés \& comme caillés des quatre humeurs, \& entre les Arabes plusieurs ont eu opinion que les animaux qu'on appelle parfaicts, peuvent estre engendrés sans l'accouplement \& mixtion du masle \& de la femelle, \& produits sans semence, \& partant croyoyent que les matrices ne sont necessaires sinon par accident ${ }^{8}$.

Montaigne, assurément, ne dédaigne pas ce procédé de juxtaposition destructrice; il en use surtout dans l'Apologie de Raymond Sebond; il y recourt, avec ironie, en divers passages de son essai antimédical. Il s'en est servi et s'en servira maintes fois encore. Mais sur la question de la semence, il s'y prend différemment. Il ne saurait s'en tenir à une simple énumération d'opinions non concordantes. Il y va pour lui, ici encore, d'une expérience personnelle, et plus précisément du rapport intime qui lie son existence à celle de son père et de ses ancêtres. Le problème physiologique de la génération de l'animal se pose pour Michel de Montaigne non dans l'abstrait, 
mais dans le cas particulier de Michel de Montaigne: il demande aux savants de lui en rendre compte, sachant par avance qu'il n'obtiendra pas satisfaction. En l'occurrence le problème de l'origine du vivant se confond avec celui de l'origine de la maladie: le père de Montaigne a souffert de la pierre, il a donc dû lui communiquer cette disposition morbide. Mais quand il a procréé son fils, le père de Montaigne n’était pas encore malade. Agrippa, nous venons de le rappeler, après avoir évoqué les apories de la philosophie sur la formation du sperme, dénonçait l'impuissance de la pensée médicale à établir une pathologie fondée sur une connaissance indiscutable des causes de la maladie. Il juxtaposait deux questions disputées. La maladie héréditaire de la pierre sera pour Montaigne l'occasion d'engager de la même manière le combat contre la présomption médicale, en alléguant la question de la génération et simultanément celle de la cause pathogène ${ }^{9}$, en les liant donc étroitement l'une à l'autre, sur un exemple fourni de première main par celui même qui tient la plume. Il fait d'une pierre deux coups. Montaigne continue à se raconter, à communiquer au lecteur une information confidentielle, et déjà ce qu'il nous dit de lui-même et de sa famille tend à mettre dans l'embarras le discours médical. L'auteur des Essais se propose lui-même, dans son expérience de la maladie, comme une énigme insoluble. Aussi peutil mêler, de façon ironique, l'autoréférence et les considérations épistémologiques les plus générales.

La stratégie de Montaigne consiste à désarmer par avance l'adversaire, et à contester la valeur des concessions que celui-ci pourrait faire. Ainsi en va-til lorsqu'un aveu d'ignorance, de la part des savants, est destiné à mieux accréditer, sur d'autres points, une certitude présomptueuse:

Il est certaine façon d'humilité subtile qui naist de la presomption, comme cette-cy, que nous reconnoissons nostre ignorance en plusieurs choses, et sommes si courtois d'avouer qu'il y a ès ouvrages de nature aucunes qualitez et conditions qui nous sont imperceptibles, et desquelles nostre suffisance ne peut descouvrir les moyens et les causes. Par cette honneste et conscientieuse declaration, nous esperons gaigner qu'on nous croira aussi de celles que nous dirons entendre ${ }^{10}$.

Notre ignorance, argumente Montaigne, ne concerne pas seulement les opérations de la nature en quelque région lointaine. Nous sommes inaptes à comprendre, au-dedans de nous, ce qui se transmet par voie d'hérédité et se trame en nos reins:

Nous n'avons que faire d'aller trier des miracles et des difficultez estrangeres; il me semble que, parmy les choses que nous voyons ordinairement, il y a des estrangetez si incompre- 
hensibles qu'elles surpassent toute la difficulté des miracles. Quel monstre est-ce, que cette goutte de semence dequoy nous sommes produits, porte en soy les impressions, non de la forme corporelle seulement, mais des pensemens et inclinations de nos peres? Cette goute d'eau où loge elle ce nombre infiny de formes?

Chemin faisant, on vient de le remarquer, Montaigne a saisi l'occasion de critiquer la notion de miracle, en faisant observer l'aspect miraculeux d'un phénomène qui appartient au «progrez ordinaire» de la nature ${ }^{12}$. Il s'agit là d'un fait admirable dont théologiens et médecins n'avaient pas manqué de débattre. Pierre Villey signale que Montaigne peut avoir lu sur le sujet un long chapitre d'Athénagoras ${ }^{13}$, ou le Traité des Monstres de Paré (XIII) et qu'il a lui-même traduit, dans le Liber Creaturarum de Sebond, les lignes suivantes: «De vray si ce n'estoit [Dieu] qui seroit-ce qui d'un si petit corps auroit fait naistre tant de parts? qui auroit multiplié une goutte d'humeur en tant de membres, de tant de formes de si diverse disposition?» ${ }^{14}$

Montaigne ne va pas en rester à cet étonnement. Il va démontrer ce qu'a été, en son propre cas, la transmission de la «forme corporelle», puis celle des «pensemens et inclinations».

La «forme corporelle»:

Il est à croire que je dois à mon pere cette qualité pierreuse, car il mourut merveilleusement affligé d'une grosse pierre qu'il avoit en la vessie; il ne s'apperceust de son mal que le soixante-septiesme an de son aage... J'estoy nay vingt cinq ans, et plus, avant sa maladie, et durant le cours de son meilleur estat [...] Où se couvoit la propension à ce deffaut? Et, lors qu'il estoit si loing du mal, cette legere piece de sa substance dequoy il me bastit, comment en portoit elle pour sa part une si grande impression? Et comment encore si couverte que quarante cinq ans apres, j'aye commencé à m'en ressentir, seul jusqu'à cette heure entre tant de freres et de sœurs, et tous d'une mere? Qui m'esclaircira de ce progrez, je le croiray d'autant d'autres miracles qu'il voudra; pourveu que comme ils font, il ne me donne pas en payement une doctrine beaucoup plus difficile et fantastique que n'est la chose mesme ${ }^{15}$.

Montaigne met la philosophie naturelle et la médecine au défi de lui donner une explication causale cohérente, objective, éclairant tous les enchaînements du «progrez» de son mal. Quant à lui, il n'est capable que de livrer une anamnèse chronologique et familiale, où domine le paradoxe apparent de la transmission d'une maladie qui ne s'était pas encore déclarée chez son père. (Il est inutile d'insister sur le fait que la génétique moderne fournirait, dans une large mesure, l'explication demandée par Montaigne, et rendrait compte de la santé mieux conservée de ses frères et sœurs indemnes de lithiase.) Montaigne offre la chose; il attend qu'on lui en fournisse, en échange («en payement»), la doctrine. Il est lui-même l'objet à expliquer, l'interpretan- 
dum. (Agrippa, parlant de la «secte dogmatique» en médecine, dit qu'«elle s'amuse plus apres les vocables et paroles qu'aux choses mesmes»... ${ }^{16}$ Et ce sera un jeu favori de Montaigne que d'opposer la chose réelle à la cause alléguée par le discours des «savants»: «Ils laissent là les choses, et s'amusent à traiter les causes. Plaisants causeurs». III, XI, p. 1026.) Il connaît la face subjective de la maladie, dans la mesure où il en vit les tourments, dans la manière dont il en est «essayé» et dont il y trouve le prétexte, en retour, d'un «essai» de ses forces morales. C'est là pour lui l'essentiel. Il ne refusera pas une explication objective, si on peut lui en offrir une qui le satisfasse. Mais il doute qu'on la trouve jamais.

De surcroît, Montaigne a hérité de son père certains «pensemens et inclinations». Lesquels? Il va nous le dire: c'est précisément la méfiance à l'égard de la médecine, le désir d'écarter toute intrusion de l'«art» dans les épreuves de la maladie:

Que les medecins excusent un peu ma liberté, car, par cette mesme infusion et insinuation fatale, j'ay receu la haine et le mespris de leur doctrine: cette antipathie que j'ay à leur art m'est hereditaire. Mon pere a vescu soixante et quatorze ans, mon ayeul soixante et neuf, mon bisayeul pres de quatre vingt, sans avoir gousté aucune sorte de medecine ${ }^{17}[\ldots]$.

L'antipathie, dans la philosophie de la renaissance, est un fait de nature. Montaigne nous laisse entendre que son refus de la médecine est aussi «fatal» que sa «forme corporelle». Il ne demande plus, cette fois, qu'on le lui explique. Il le mentionne comme une donnée irrécusable, liée à son individualité propre et à celle de la lignée dont il descend. Or ce fait va de pair avec un autre fait: la très satisfaisante longévité du père, de l'aïeul, et du bisaïeul. Ainsi liés, ces deux faits peuvent être considérés comme une expérience, qu'il est légitime d'opposer à celle dont se prévaut la médecine: «La medecine se forme par exemples et experience; aussi fait mon opinion.» ${ }^{18}$ Peu importe si Montaigne, lui, est destiné à vivre moins longtemps...

Ainsi, l'on constate qu'au moment où il engage le débat avec la médecine, Montaigne choisit de se retrancher dans la certitude particulière dont il peut être le garant absolu. Le voici, tel que son père l'a formé, souffrant de la pierre et réfractaire aux «promesses» de la médecine. Serait-il légitime de dire qu'il subjectivise les problèmes dont la médecine veut traiter de manière objective, mais où elle aboutit, selon Agrippa, à d'indépassables contradictions? Je ne crois pas que l'on puisse parler ici de subjectivation. La stratégie de Montaigne consiste à se désigner lui-même, jusque dans son expérience subjective, comme un objet réfractaire à toutes les entreprises du discours 
médical. Tout se passe comme s'il disait aux médecins: «Je suis la question que vous ne pouvez résoudre. Avant de prétendre guérir mon mal, expliquezmoi à moi-même». On parlerait aussi bien ici d'une objectivation de l'expérience personnelle, destinée à constituer l'obstacle évident et vivant, la pierre d'achoppement, opposés à la présomption du savoir ${ }^{19}$.

Assurément, lorsqu'il attribue à ses opinions une origine «fatale», Montaigne sait bien qu'il les fonde en nature, mais qu'il leur retire tout véritable fondement rationnel. Or, quelle que soit sa préférence pour ce que lui dicte la nature, Montaigne ne renonce pas à poursuivre, contre la médecine, une discussion fondée en raison. Il prend donc soin d'ajouter:

Il est possible que j'ay receu d'eux [mes ancêtres] cette dispathie naturelle à la médecine; mais s'il n'y eut eu que cette consideration, j'eusse essayé de la forcer. Car toutes ces conditions qui naissent en nous sans raison, elles sont vitieuses, c'est une espece de maladie qu'il faut combattre; il peut estre que j'y avois cette propension, mais je l'ay appuyée et fortifiée par les discours qui m'en ont estably l'opinion que j'en ay ${ }^{20}$.

La suite de l'essai, bien que Montaigne y fasse constamment appel à son expérience propre, sera consacrée à l'exposé de ces raisonnements (qu'il nomme «discours», selon la langue de l'époque). Il pourra y insérer bien des arguments formulés par Agrippa, en les transportant cette fois plus directement dans son propre texte. L'emprunt, dès lors, est plus visible, parce qu'il se formule sur le même mode impersonnel et général, parce qu'il recourt aux mêmes exemples historiques, aux mêmes autorités, à la même méthode doxographique. Mais dans le cas de la semence et de la cause des maladies, Montaigne aura été tout ensemble fidèle à l'ordre des matières traitées par Agrippa, et profondément original. Tout porte à croire qu'il a suivi, sur ce point, mais selon le mode de la lecture appropriative dont il se réclamait, le dispositif d'attaque adopté par Agrippa. Jusqu'aux problèmes qui concernent la validité du savoir médical, Montaigne reste celui qui a déclaré, dès son adresse Au Lecteur: «Je suis moy-mesmes la matiere de mon livre.» ${ }^{21} \mathrm{Il}$ a «transplanté» non seulement dans son livre, mais en lui-même, les thèses et les exemples «paradoxaux» de son prédécesseur.

\section{Notes}

1 Pierre Villey, Les sources et l'évolution des Essais de Montaigne. 2 vol., Paris 1908.

${ }^{2}$ Les Essais de Michel de Montaigne, édition municipale, t. IV: Les sources des Essais. Annotations et éclaircissements par Pierre Villey. Bordeaux 1920. 
${ }^{3}$ Francesco Petrarca, Invective contra medicum [...] edizione critica a cura di Pier Giorgio Ricci. Appendice di aggiornamento a cura di Bortolo Martinelli. Roma 1978.

${ }^{4}$ Les Essais de Michel de Montaigne, éd. Pierre Villey. Rééd. et préface de V. L. Saulnier. PUF, Paris, et Guilde du Livre, Lausanne 1965, Livre II, xxxvii, p. 771.

${ }^{5}$ Op.cit., p. 759.

${ }^{6}$ Op.cit., p. 760.

7 Op.cit., p. 762.

${ }^{8}$ Agrippa a lui-même repris l'information fournie par Plutarque, Opinions des philosophes, V, iii. Nous citons d'après la traduction de Louis Turquet de Mayerne, parue chez Jean Durand, s.l., en 1582. C'est la première édition d'une traduction française de cet ouvrage dont le texte latin, paru d'abord en 1526-1527, avait été maintes fois réimprimé. Rappelons le titre complet, où s'exprime le caractère fidéiste de ce livre: De incertitudine et vanitate scientiarum et artium atque excellentia verbi dei declamatio. Le passage cité se trouve aux pages 416-4.17 de l'édition française.

Ce passage sur la génération et la semence a été transcrit presque littéralement par Montaigne dans l'Apologie de Raymond Sebond, p.556-557. Il ne peut désormais plus le réutiliser de la même manière.

${ }^{9}$ Montaigne reprendra plus loin, p. 771, la question de l'embarras des médecins sur l'étiologie des maladies. Son texte, alors, suivra de très près celui d'Agrippa sur le même problème (p.417 de l'édition française). Les «opinions» successivement citées seront exactement les mêmes.

${ }^{10}$ Op.cit., p. 762.

${ }^{11}$ Ibid.

${ }^{12}$ On sait que Montaigne a raisonné de la même manière en ce qui concerne les monstres. Cf. H.Debrunner, «Michel de Montaigne und die Lehre von den Missbildungen», Gesnerus 3 (1946) fasc. 1, p.1-7.

${ }^{13}$ De resurrectione mortuorum, 17. Le texte a été traduit par Arnaud du Ferrier, chez Simon Millanges, Bordeaux 1577.

${ }^{14}$ La Théologie Naturelle de Raymond Sebon, 2 vol., in CEuvres complètes de Michel de Montaigne, éd. Armaingaud, IX et X. Paris 1932, t. IX (1 $1^{\text {re }}$ partie), chap.LVII, p.92. Sur l'héritage de la pierre et le rapport de Montaigne à son père, cf. Antoine Compagnon, Nous, Michel de Montaigne, Paris 1980, passim.

${ }^{15}$ Op.cit., p. 763-764.

${ }^{16}$ De l'incertitude [...], p.414.

17 Op.cit., p. 764.

18 Ibid.

19 Tout ce que Montaigne consent à retenir de la médecine traditionnelle, c'est l'hygiène: la «forme de vivre» (p.767), ou le «régime de vivre» (p.775). Encore, sur ce point, estime-t-il préférable de ne pas suivre les avis contradictoires des médecins (qu'il évoque p. 775), mais de se fier à son propre appétit. Dans notre récent ouvrage, Montaigne en mouvement (Gallimard, Paris 1982), nous avons essayé de montrer que Montaigne, dans l'essai final (III, xiii, De l'experience), donnait en exemple sa propre pratique des «six choses nonnaturelles». S'il a exposé une fois un «programme» medical (II, xxxvii, p. 773), celui-ci ne comporte rien de novateur: c'est celui qu'établissait la médecine de son temps, et qu'on peut trouver notamment dans l'œuvre de Fernel. Montaigne tient à nous persuader que le 
médecin est inévitablement conduit à se tromper (à se «mesconter»), en raison même de la multiplicité des facteurs que le discours médical institué déclare déterminants et oblige à prendre en considération. - Au demeurant, la polémique antimédicale de Montaigne reste courtoise, en comparaison des invectives ordurières d'Agrippa.

20 Op.cit., p. 765.

${ }^{21}$ Op.cit., p.3.

\section{Résumé}

L'attaque d'Agrippa de Nettesheim contre la médecine commence par mettre en cause l'ignorance des savants sur l'origine de la semence. Montaigne, qui a utilisé ce texte d'Agrippa dans l'Apologie de Raymond Sebond, suit à nouveau la stratégie antimédicale de son modèle dans l'essai II, xxxvii. Cette fois, il prend pour exemple la lithiase urinaire qu'il a héritée de son père. L'argument tend à prouver que la médecine ne peut rendre compte de la génération et de la transmission des caractères morbides.

\section{Summary}

In his De incertitudine et vanitate scientiarum, Agrippa von Nettesheim opens his attack on medicine in accumulating the contradictory opinions of the philosophers about the formation of semen. Not content to transcribe Agrippa's lines on the subject in the Apologie de Raymond Sebond (II, xii), Montaigne relies on the same antimedical strategy in his essay De la ressemblance des enfans aux peres (II, xxxvii), in which he describes his own inherited urinary stone disease. Asking for an explanation which the doctors are unable to give him, Montaigne, like Agrippa, makes the semen issue his first argument against medicine. In this occasion, the case against medicine is handled differently, as Montaigne feels personnally involved.

Prof. Dr. Jean Starobinski

12 , rue de Candolle

CH-1200 Genève 